\title{
THE STABLE NEIGHBORHOOD THEOREM AND LENGTHS OF CLOSED GEODESICS
}

\author{
ARA BASMAJIAN \\ (Communicated by Clifford J. Earle, Jr.)
}

\begin{abstract}
We derive a generalized collar lemma, called the stable neighborhood theorem, for nonsimple closed geodesics. As an application, we show that there is a lower bound for the length of a closed geodesic having crossing number $k$ on a hyperbolic surface. This lower bound only depends on $k$ and tends to infinity as $k$ goes to infinity. Also, we show that the shortest nonsimple closed geodesic on a closed hyperbolic surface has (geometric) crossing number bounded above by a constant which only depends on the genus.
\end{abstract}

\section{INTRODUCTION}

It is well known that a simple closed geodesic on a hyperbolic surface has a collar neighborhood whose width only depends on the length of the geodesic $[\mathrm{Bu}$, $\mathrm{Ha}, \mathrm{K}, \mathrm{M}, \mathrm{Ma}, \mathrm{R}]$. In this paper, we derive a type of generalized collar lemma (the stable neighborhood theorem) for closed geodesics with self-intersection. The notion of a collar is replaced by the concept of a stable neighborhood about a closed geodesic. The stable neighborhood theorem says that a closed geodesic on a hyperbolic surface has a stable neighborhood whose width only depends on the length of the closed geodesic.

Hempel, Nakanishi, and Yamada [He, N, Y1, Y2] have shown that there is a universal lower bound for the length of a nonsimple closed geodesic on a hyperbolic surface. As a consequence of the stable neighborhood theorem, we show that in fact one can improve this lower bound (Corollary 1.2) by knowing the crossing number of the closed geodesic.

Finally, using a theorem of Bers on pants decompositions of Riemann surfaces, we show that the number of crossings of the shortest closed geodesic with at least $k$-fold crossings on a closed hyperbolic surface is bounded from above by a constant depending only on $k$ and the genus of the surface.

Received by the editors May 30, 1991 and, in revised form, January 31, 1992.

1991 Mathematics Subject Classification. Primary 30F35.

Key words and phrases. Closed geodesics, collar, hyperbolic surface, self-intersection number.

This paper was written while the author was a visiting research scholar at Stony Brook University in the spring semester of 1991. The author would like to thank the Department of Mathematics at Stony Brook University, especially Irwin Kra and Bernard Maskit, for the invitation. 


\section{Statement of Results}

The neighborhood of width $d, U_{d}(\omega)$, about a geodesic $\omega$ in a hyperbolic surface is the set of all points within a distance $d$ from the geodesic. Suppose $\omega$ is a closed geodesic. Then the neighborhood $U_{d}(\omega)$ is said to be stable if for any two connected (smooth) lifts of $\omega$, say $\omega_{1}$ and $\omega_{2}$, we have

$$
\omega_{1} \cap \omega_{2} \neq \varnothing \quad \text { if and only if } U_{d}\left(\omega_{1}\right) \cap U_{d}\left(\omega_{2}\right) \neq \varnothing \text {. }
$$

Clearly a closed geodesic has stable neighborhoods for sufficiently small widths (which depend on the hyperbolic structure of the surface). Our interest is in finding stable neighborhoods whose widths depend only on the length of the geodesic and not on the underlying hyperbolic structure of the surface.

We associate to each closed geodesic $\omega$ of length $\ell=\ell(\omega)$ the function,

$$
c(\omega)=c(\ell)=\log \operatorname{coth}(\ell / 4) \text {. }
$$

Theorem 1.1 (The stable neighborhood theorem). A closed geodesic of length $\ell$ on a hyperbolic surface has a stable neighborhood of width $c(\ell)$. Furthermore, two disjoint closed geodesics $\omega_{1}$ and $\omega_{2}$ on the surface having lengths $\ell_{1}$ and $\ell_{2}$ have disjoint stable neighborhoods of widths $c\left(\ell_{1}\right)$ and $c\left(\ell_{2}\right)$, respectively, if $\omega_{1}$ and $\omega_{2}$ are separated by a disjoint union of simple closed geodesics.

We remark that the separation condition in the first part of the theorem is necessary. For example, a pair of pants has closed geodesics that get arbitrarily close to any fixed boundary geodesic. Of course, the crossing numbers of these closed geodesics get arbitrarily large as they get closer to the boundary geodesic. Thus the width of the stable neighborhood must take into account the crossing number of the other closed geodesic.

Corollary 1.2. There exists an increasing sequence $M_{k}$ (for $\left.k=1,2,3, \ldots\right)$ tending to infinity so that if $\omega$ is a closed geodesic with self-intersection number $k$, then $\ell(\omega)>M_{k}$. Thus the length of a closed geodesic gets arbitrarily large as its self-iniersection gets large.

It is well known that the shortest closed geodesic on a hyperbolic surface which is not a thrice punctured sphere is simple. In other words, it has crossing number zero. Furthermore, the length of the shortest closed geodesic on a hyperbolic surface of genus $g$ is bounded from above by a constant which only depends on the genus of the surface (see [Ber]). Following these lines, we let $s_{k}(S)$ be the length of the shortest closed geodesic with at least $k$-fold intersection on the hyperbolic surface $S$. We are interested in upper bounds (depending only on topological data) for the lengths of these short geodesics.

Proposition 1.3. There are constants $N_{k}(g)$, depending only on the crossing number of the geodesic and the genus of the surface, so that the shortest closed geodesic with at least $k$-fold intersection on a surface of genus $g$ has length bounded from above by $N_{k}(g)$.

Peter Buser has asked whether the shortest nonsimple closed geodesic on a hyperbolic surface has only one self-intersection. As an elementary consequence of Corollary 1.2 and Proposition 1.3, we have

Corollary 1.4. Suppose $\gamma_{S}$ is the shortest closed geodesic with crossing number at least $k$ on the hyperbolic surface $S$ of genus $g$. Then the crossing number 
of $\gamma_{S}$ is bounded from above by a constant which only depends on $k$ and $g$. Of course, for $k=0$ we know that the upper bound can be taken to be zero.

The author wishes to thank Peter Buser, Jozef Dodziuk, Darryl McCullough, Burton Randol, and Mark Thornber for helpful suggestions in the preparation of this manuscript, and also the referee for pointing out that the original definition of stable neighborhood was not correct.

\section{BASICS AND NOTATION}

The reader is referred to the paper [Ba] for most of the basic definitions involved. In this paper, we use $d(\cdot, \cdot)$ to denote hyperbolic distance in the hyperbolic plane.

In order to prove the above theorems we need to define some words. A fuchsian group is a discrete subgroup of orientation preserving isometries of the hyperbolic plane $\mathbb{H}^{2}$. The quotient of the hyperbolic plane by a fuchsian group is a hyperbolic surface. A hyperbolic surface (and the group representing it) is said to be a pair of pants if it is topologically a thrice-punctured sphere.

Let $\mathbb{T}^{1}$ be the unit circle and suppose $\omega: \mathbb{T}^{1} \rightarrow S$ is a (parametrized) closed geodesic on the hyperbolic surface $S$. Define the crossing (or self-intersection) number of $\omega$ to be the number of transverse intersections that $\omega$ makes with itself.

The action of the isometries on the hyperbolic plane extends to the circle at infinity. An isometry having two fixed points on the circle at infinity is known as a hyperbolic element. The axis of a hyperbolic element is the unique geodesic in the hyperbolic plane joining the fixed points. We use $A(\gamma)$ to denote the axis of $\gamma$. The translation length of $\gamma$, denoted $\ell(\gamma)$, is the hyperbolic distance that a point on $A(\gamma)$ is moved. We will use the same notation for the length of a closed geodesic on the surface.

Suppose $\gamma$ and $\beta$ are hyperbolic elements having disjoint axes. We say that $\gamma$ and $\beta$ are standard generators for a pair of pants $G$ if

(1) $\gamma$ and $\beta$ generate a torsion-free fuchsian group which represents a pair of pants;

(2) after possibly changing the orientations of $\gamma$ and $\beta$ so that their axes are oriented to the right of each other, $\gamma, \beta$, and $\beta \gamma$ are primitive boundary elements of $G$, each representing different boundary components of the pair of pants.

The main tool in the following arguments is the pair of pants theorem [Ba], which states that $\gamma$ and $\beta$ are standard generators for a pair of pants if and only if $c(\gamma)+c(\beta) \leq d$, where $d$ is the distance from the axis of $\gamma$ to the axis of $\beta$.

\section{The PRoofs of Theorem 1.1 AND Corollary 1.2}

In order to prove Theorem 1.1 we need the following lemma, which appears in the proof of a proposition in [GM, p. 19].

Lemma 3.1. Two hyperbolic elements with equal translation lengths having disjoint axes in a torsion-free fuchsian group form standard generators for a pair of pants. 


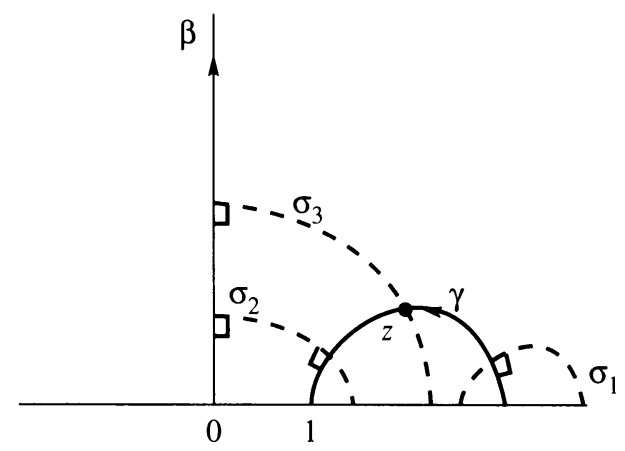

FIGURE 1

For the reader's convenience, we supply the proof.

Proof. Without loss of generality, we can normalize the hyperbolic elements and call them $\beta$ and $\gamma$, as in Figure 1. Now, as usual, embed the group $\langle\beta, \gamma\rangle$ as an index two subgroup of the reflection group $\left\langle\sigma_{1}, \sigma_{2}, \sigma_{3}\right\rangle$. Thus $\gamma=\sigma_{2} \sigma_{1}$ and $\beta=\sigma_{3} \sigma_{2}$. The pair $(\gamma, \beta)$ will be standard generators for a pair of pants if and only if the $\sigma_{i}$-reflection circles bound a free polygon (see [Ba] for the details).

If $\beta A(\gamma) \cap A(\gamma) \neq \varnothing$, this means that the $\sigma_{3}$-reflection circle must intersect $A(\gamma)$. Then either the $\sigma_{3}$-reflection separates $\sigma_{2}$ from $\sigma_{1}$ or the $\sigma_{1}$ reflection circle intersects $A(\beta)$. Note that the reflection circles cannot intersect since this would introduce torsion into the original group. Let $z$ be the intersection point between $A(\gamma)$ and the $\sigma_{3}$-reflection circle. Now, if the $\sigma_{3}$-reflection circle separates then (Figure 1)

$$
\frac{\ell(\beta)}{2}=d\left(\sigma_{2}, \sigma_{3}\right)<d\left(\sigma_{2}, z\right)<d\left(\sigma_{2}, \sigma_{1}\right)=\frac{\ell(\gamma)}{2},
$$

where the first inequality follows from the fact that the common orthogonal between two geodesics realizes the minimum distance between any two points on the geodesics. This clearly contradicts our assumption that $\ell(\gamma)=\ell(\beta)$. If, on the other hand, the $\sigma_{1}$-reflection circle intersects $A(\beta)$ then the same argument as above works by considering the distance $\frac{\ell(\gamma)}{2}=d\left(\sigma_{1}, \sigma_{2}\right)$. Thus the $\sigma_{i}$-reflection circles must bound a free polygon and hence are standard generators for a pair of pants.

Suppose $\omega$ is a closed geodesic on the hyperbolic surface $S$. Fix a lift $\beta$ of the geodesic $\omega$. Consider the region $\mathscr{R}$ of points in the hyperbolic plane that are a distance less than $c(\omega)$ from the axis $A(\beta)$. That is,

$$
\mathscr{R}=\left\{z \in \mathbb{H}^{2} \mid d(z, A(\beta))<c(\omega)\right\} .
$$

We claim that this projects to $S$ as a stable neighborhood of $\omega$.

Proof of Theorem 1.1. Let $G$ be the fuchsian group representing the hyperbolic surface $S$. We first would like to show that $\omega$ has a stable neighborhood of width $c(\omega)$. Consider the region $\mathscr{R}$ constructed above and suppose that 
$g \mathscr{R} \cap \mathscr{R} \neq \varnothing$ for some $g \in G$. We claim that $g A(\beta) \cap A(\beta) \neq \varnothing$. To see this, consider the hyperbolic elements $\beta$ and $g \beta g^{-1}$ and suppose that the $g$-translate of $A(\beta)$ does not cross $A(\beta)$. Then since the translation length of $\beta$ equals the translation length of its conjugate $g \beta g^{-1}$, by Lemma 3.1, $\left(\beta, g \beta g^{-1}\right)$ are standard generators for a pair of pants. Hence

$$
2 c(\omega)=c(\beta)+c\left(g \beta g^{-1}\right) \leq d\left(A(\beta), A\left(g \beta g^{-1}\right)\right),
$$

contradicting our assumption that $g \mathscr{R} \cap \mathscr{R} \neq \varnothing$. Thus, $g A(\beta) \cap A(\beta) \neq \varnothing$. This implies that the region $\mathscr{R}$ projects to the desired stable neighborhood.

Now, suppose $\omega_{1}$ and $\omega_{2}$ are disjoint closed geodesics which are separated by simple closed geodesics. If the stable neighborhoods of these geodesics overlap, then we can find lifts $\gamma$ and $\beta$ to the fuchsian group $G$ so that the length $d$ of the common orthogonal between them satisfies $d<c(\gamma)+c(\beta)$. On the other hand, since the orthogonal from the axis $A(\gamma)$ to the axis $A(\beta)$ must intersect the axis of a simple hyperbolic in $G$, it must be that $\beta A(\gamma) \cap A(\gamma)=\varnothing$ and $\gamma A(\beta) \cap A(\beta)=\varnothing$. This implies that $(\gamma, \beta)$ are standard generators for a pair of pants and hence, by the pair of pants theorem, $c(\gamma)+c(\beta) \leq d$. This is clearly a contradiction.

Before we prove Corollary 1.2, we need a couple of lemmas.

Lemma 3.2. There exists a decreasing function $A=A(\ell)$ depending only on $\ell$ and tending to zero so that if $\omega$ is any closed geodesic on a hyperbolic surface of length $\ell$, then any geodesic loop contained in $\omega$ has length bigger than $A$. In other words, any segment in $\omega$ of length less than or equal to $A(\ell)$ is embedded.

Proof. Suppose $\omega_{1}$ is a geodesic loop in $\omega$, and let $\omega_{2}$ be the other geodesic loop so that $\omega=\omega_{1} \cup \omega_{2}$. Using Theorem (8.3.1) of [Bea] we have

$$
\ell\left(\omega_{1}\right) \geq 2 \sinh ^{-1}\left(\frac{1}{\sinh \ell\left(\omega_{2}\right) / 2}\right)>2 \sinh ^{-1}\left(\frac{1}{\sinh \ell / 2}\right),
$$

where the last inequality follows from the fact that $\omega_{2}$ is shorter than $\omega$. Setting $A$ to be this last term yields the lemma.

Lemma 3.3. Suppose $\beta$ is a closed geodesic of length $\ell$ on a hyperbolic surface $S$ having at least one self-intersection. There exists a decreasing function $B=$ $B(\ell)$, depending only on $\ell$ and tending to zero so that if $I$ and $J$ are any two embedded compact segments in $\beta$ having disjoint interiors and common endpoints, then either

$$
\ell(I)>B \text { or } \ell(J)>B .
$$

Proof. Set $S=\mathbb{H}^{2} / G$ and label the common endpoints $z$ and $w$. Observe that $I$ and $J$ cannot meet smoothly at both $z$ and $w$, since that would force the geodesic $\omega$ to be simple. Also, if $I$ and $J$ meet smoothly at one point and not the other then $I \cup J$ is a loop in $\omega$ and hence by Lemma 3.2 either $\ell(I)>A / 2$ or $\ell(J)>A / 2$. Thus set $B$ to be $A / 2$ in this case.

From now on assume that the intersections at $z$ and $w$ are not smooth. Then in $\mathbb{H}^{2}$, there exists a triple of distinct geodesics $\left(L_{1}, L_{2}, L_{3}\right)$ each one the 
axis of a hyperbolic element in $G$ representing $\beta$ (these elements are conjugate in $G$ ) satisfying:

(1) $L_{1} \cap L_{2}=z_{1}$ is a lift of $z$.

(2) $L_{2} \cap L_{3}=w_{1}$ is a lift of $w$ with the line segment from $z_{1}$ to $w_{1}$ covering $J$.

(3) There exists a point $w_{2} \in L_{1}$ so that the line segment from $z_{1}$ to $w_{2}$ covers $I$.

(4) There exists $g \in G$ taking $L_{3}$ to $L_{1}$ with $g\left(w_{1}\right)=w_{2}$.

Now if $L_{1} \cap L_{3}=\varnothing$, then we have

$$
\ell(J)=d\left(z_{1}, w_{1}\right) \geq s \geq 2 c(\ell),
$$

where $s$ is the orthogonal distance from $L_{2}$ to $L_{3}$ and the last inequality follows from the stable neighborhood theorem.

Next if $L_{1} \cap L_{3} \neq \varnothing$, let $x$ be this intersection point. There are two cases to consider.

The first case is if the distances $d\left(x, z_{1}\right)$ and $d\left(x, w_{1}\right)$ are both less than or equal to $A / 4$, where $A$ is the constant in Lemma 3.2. Since the segment from $x$ to $g(x)$ forms a loop in $\beta$ by Lemma 3.2, we have

$$
\begin{aligned}
A & \leq d(x, g(x)) \leq d\left(x, z_{1}\right)+d\left(z_{1}, g\left(w_{1}\right)\right)+d\left(g\left(w_{1}\right), g(x)\right) \\
& \leq A / 4+A / 4+d\left(z_{1}, g\left(w_{1}\right)\right) .
\end{aligned}
$$

Hence, we can conclude that $\ell(I) \geq A / 2$.

The other case to consider is that either the distance $d\left(x, z_{1}\right)>A / 4$ or $d\left(x, w_{1}\right)>A / 4$. First, observe that Theorem 11.6 .8 of [Bea] says that there is a positive lower bound to the angle of intersection at $x$ between $L_{1}$ and $L_{3}$; moreover, this lower angle bound only depends on $\ell$, the length of $\beta$. Now in either case, using the appropriate hyperbolic sine rule coupled with our lower angle bound yields a lower bound $D=D(\ell)$ for either $\ell(I)$ or $\ell(J)$.

To finish the proof, set $B=\min (A / 2,2 c(\ell), D)$ and note that $B$ is decreasing since $A(\ell), c(\ell)$, and $D(\ell)$ are decreasing functions of $\ell$.

Proof of Corollary 1.2. Suppose $\omega$ has crossing number $k$. Cut $\omega$ into $n$ segments each of length $B(\ell) / 2$ except possibly for one segment whose length is smaller than $B(\ell) / 2$. In any event,

$$
n \leq 2 \frac{\ell}{B(\ell) / 2}
$$

If $k<n$, then applying inequality (3.4) we get that

$$
\ell>\left(\frac{k}{4}\right) B(\ell) .
$$

Next suppose $k \geq n$. Then there must be a segment containing $k / n$ crossings. Since, by Lemma 3.3, each crossing in this segment contributes at least $B(\ell) / 2$, we have

$$
\ell>\frac{k}{n}\left(\frac{B(\ell)}{2}\right) .
$$


Using inequality (3.4) and solving the above inequality for $\ell$ we have

$$
\ell>\sqrt{\frac{k}{2}}\left(\frac{B(\ell)}{2}\right) .
$$

Finally, let $x_{k}$ and $y_{k}$ be the unique fixed points of the right-hand sides of (3.5) and (3.6), respectively. Setting $M_{k}=\min \left(x_{k}, y_{k}\right)$, it is easy to see that this sequence is increasing and tends to infinity with $k$.

\section{The Proofs of Proposition 1.3 ANd Corollary 1.4}

The space $\mathscr{M}$ of all hyperbolic metrics (structures) on a sphere with three holes can be parametrized by three nonnegative real numbers which describe the lengths of the three ordered boundary geodesics (see Abikoff [A] for the particulars). If $\omega$ is a free homotopy class of a closed curve on a hyperbolic surface $S$, we will denote the length of the geodesic in the free homotopy class of $\omega$ with respect to the $S$-metric by $\ell_{S}(\omega)$. We will say that a pair of pants is $\varepsilon$-thin if the lengths of all three of its boundary geodesics are at most $\varepsilon$. We first need the following lemma about $\varepsilon$-thin pants.

Lemma 4.1. Let $\omega$ be a closed curve on a sphere with three holes. Then the geodesic length function $\ell_{P}(\omega)$ is a continuous function of $\mathscr{M}$ and, furthermore, given $\varepsilon>0$ there is a constant $B(\varepsilon, \omega)$, depending only on $\varepsilon$ and the free homotopy class of $\omega$, so that

$$
\ell_{P}(\omega) \leq B(\varepsilon, \omega)
$$

for all $\varepsilon$-thin pairs of pants $P$.

Proof. Let $\gamma_{1}, \gamma_{2}$, and $\gamma_{3}$ be the boundary curves on a sphere with three holes. The space of hyperbolic structures $\mathscr{M}$ (note that we include punctures) can be parametrized by

$$
\mathscr{M}=\left\{\left(\ell_{1}, \ell_{2}, \ell_{3}\right) \in R_{+}^{3} \cup\{0\} \mid \ell\left(\gamma_{1}\right)=\ell_{1}, \ell\left(\gamma_{2}\right)=\ell_{2}, \ell\left(\gamma_{3}\right)=\ell_{3}\right\} .
$$

It is well known that the geodesic length function $\ell_{P}(\omega)$ is a continuous function on $\mathscr{M}$. The set of $\varepsilon$-thin pairs of pants form the subset

$$
T_{\varepsilon}=\left\{\left(\ell_{1}, \ell_{2}, \ell_{3}\right) \in R_{+}^{3} \cup\{0\} \mid \ell_{1}, \ell_{2}, \ell_{3} \leq \varepsilon\right\} .
$$

$T_{\varepsilon}$ is clearly compact in $\mathscr{M}$, and hence $\ell_{P}(\omega)$ has an upper bound as desired.

Proof of Proposition 1.3. Suppose $S$ is a closed surface. Bers [Ber] showed that there exists a constant depending only on the genus, so that any surface of genus $g$ has a pants decomposition where the lengths of all the boundary geodesics of the pairs of pants are bounded by this constant. We call this constant the Bers constant and denote it by $L_{g}$.

Using the Bers constant, it is elementary to see that there is an upper bound on $s_{k}(S)$, depending only on the genus of the surface; namely, for each hyperbolic surface there is an embedded $L_{g}$-thin pair of pants. Pick a free homotopy class $\omega$ having $k$-fold intersection number in a sphere with three holes and observe that its length on an $L_{g}$-thin pair of pants, by Lemma 4.1, is bounded by a constant depending only on $L_{g}$ and, hence, only on the genus of the surface. 
Proof of Corollary 1.4. Suppose $\gamma_{S}$ is the shortest closed geodesic with intersection number at least $k$ on the hyperbolic surface $S$ of genus $g$. We have seen, by Proposition 1.3, that there is an upper bound $N_{k}(g)$ so that

$$
\ell_{S}\left(\gamma_{S}\right) \leq N_{k}(g) \text {. }
$$

Now, we also know from Corollary 1.2 that the length of $\gamma_{S}$ gets arbitrarily large with its self-intersection number. Putting this fact together with inequality $(*)$ tells us that there is an upper bound, which only depends on $k$ and $g$, to the number of self-intersections of $\gamma_{S}$. In fact, the number

$$
n(k, g)=\inf \left\{j \in \mathbb{Z}^{+}: M_{j}>N_{k}(g)\right\}
$$

serves as an upper bound, where the $M_{j}$ 's are the constants in Corollary 1.2.

\section{REFERENCES}

[A] William Abikoff, The real analytic theory of Teichmüller space, Lecture Notes in Math., vol. 820, Springer-Verlag, New York, 1980.

[Ba] Ara Basmajian, Constructing pairs of pants, Ann. Acad. Sci. Fenn. Ser. AI Math. 15 (1990), 65-74.

[Bea] Alan Beardon, The geometry of discrete groups, Springer-Verlag, New York, 1983.

[Ber] Lipman Bers, An inequality for Riemann surfaces, Differential Geometry and Complex Analysis, H. E. Rauch Memorial volume (Isaac Chavel and Herschel M. Farkas, eds.), Springer-Verlag, New York, 1985, pp. 87-93.

[Bu] Peter Buser, The collar theorem and examples, Manuscripta Math. 25 (1978), 349-357.

[GM] Jane Gilman and Bernard Maskit, An algorithm for 2-generator Fuchsian groups, Michigan Math. J. 38 (1991), 13-32.

[Ha] Naomi Halpern, A proof of the collar lemma, Bull. London Math. Soc. 13 (1981), 141-144.

[He] John Hempel, Traces, lengths, and simplicity of loops on surfaces, Topology Appl. 18 (1984), 153-161.

[K] Linda Keen, Collars on Riemann surfaces, Discontinuous Groups and Riemann Surfaces, Ann. of Math. Stud., no. 79, Princeton Univ. Press, Princeton, NJ, 1974, pp. 263-268.

[M] Bernard Maskit, Comparison of hyperbolic and extremal lengths, Ann. Acad. Sci. Fenn. Ser. AI Math. 10 (1985), 381-386.

[Ma] Peter Matelski, A compactness theorem for Fuchsian groups of the second kind, Duke Math. J. 43 (1976), 829-840.

[N] Toshihiro Nakanishi, The lengths of the closed geodesics on a Riemann surface with selfintersection, Tohoku Math. J. (2) 41 (1989), 527-541.

[R] Burton Randol, Cylinders in Riemann surfaces, Comment. Math. Helv. 54 (1979), 1-5.

[Y1] Akira Yamada, On Marden's universal constant of Fuchsian groups, Kodai Math. J. 4 (1981), 266-277.

[Y2] , On Marden's universal constant of Fuchsian group. II, J. Analyse Math. 41 (1982), 234-248.

Department of Mathematics, University of Oklahoma, Norman, Oklahoma 73019

E-mail address: abasmajian@nsfuvax.math.uoknor.edu 